vent introduction of the disease in imported dogs and cats unless highly irresponsible people smuggle their pets into Britain. ${ }^{1}$ But people visiting the many countries throughout the world where rabies is enzootic may be bitten by a rabid animal. The first sign of the disease in an animal is often a change in behaviour. Thus a "tame" or an oddly behaving wild animal may be rabid and its bite infective. Anyone bitten by any animal overseas should immediately seek local medical advice and receive treatment if necessary. On return to Britain he should immediately report to his doctor and give him a full history. There have been at least eight fatal cases of human rabies in this country in the last 25 years-all of them infected outside Britain, seven by rabid dogs. ${ }^{2}$

Any patient with an unusual encephalitis or a disease suggestive of rabies should be asked whether he has been overseas during the previous year and whether there is any history of an animal bite. Incubation periods of over one year have been reported. ${ }^{3}$ Though the full-blown disease may be clinically recognizable, at least to those who have considered the diagnosis, it may present as an encephalitis with no special neurological features and may even resemble poliomyelitis. There is no very satisfactory method for virological diagnosis during life. It may be possible to isolate and identify rabies virus from the saliva or other secretions, but this is too timeconsuming to be completed before the death of the patient in most instances, and not all infected people (or animals) secrete virus in the saliva.

It is generally accepted that the infection spreads along nerves from the bite wound to the central nervous system, and it then appears to spread from there along nerves to infect secretory glands and also the cornea. L. G. Schneider ${ }^{4}$ observed in experimentally infected mice that rabies antigen could be detected in corneal cells before the onset of clinical signs. This potentially valuable method of diagnosis during life has not yet been widely applied in naturally infected animals or man, but its successful use has just been reported from Mexico. ${ }^{5}$ A child died some 60 hours after impression smears from the cornea had been obtained and had shown rabies antigen after fluorescent antibody staining. Thus at least when it is positive (and suitable controls are used) this test offers a diagnostic method taking only about quarter of an hour in suitably equipped and experienced hands. Though further evaluation in a substantial number of cases is clearly required, the test might become important if antiviral treatment were developed. In the case reported the child was given a synthetic interferon inducer without effect, but in this almost uniformly lethal disease interferon inducers and other compounds possibly effective against RNA-viruses may be worthy of trial.

Normally the virological diagnosis of rabies is made in man or animals only at necropsy by examination of the brain. The methods are well developed and reliable. ${ }^{6}$ The fluorescent antibody staining method ${ }^{7}$ applied to impression smears from suitable areas of the brain can provide a diagnosis well within 24 hours. These findings can be confirmed by histological staining for Negri bodies in smears or sections, but the reliability of the fluorescent antibody method in experienced hands is now such that some laboratories are tending to eliminate the second step. Complete confirmation is obtained by inoculation of mice and subsequent examination of the brains of sick animals, but this requires two to three weeks. It is sometimes of value to examine the virus strain isolated by sensitive antibody-neutralization tests, as these may show small strain differences of epidemiological importance. Advice on diagnosis and also on the prophylactic treatment of human rabies can be obtained from the Virus Reference Laboratory, Central Public Health Laboratory, Colindale, London, N.W.9.

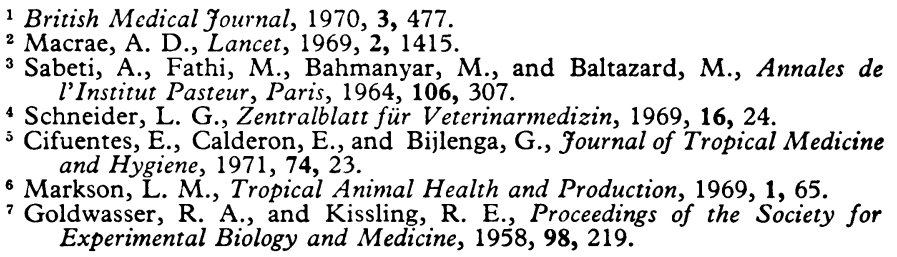

\section{Population: A Problem?}

Whether or not the Government sets up a special office to advise on population policy, some good may come of the House of Commons select committee's report ${ }^{1}$ on population published last week. Throughout its 324 pages ran one theme agreed by all who gave evidence: contraceptive advice is not yet readily enough available in Britain.

Nearly 1,000,000 children are born each year in the United Kingdom and of these $20 \%-25 \%$ are in some sense unplanned at the moment of conception. ${ }^{1}$ Perhaps 200,000 "unwanted" babies are born each year-though many become loved and cherished by either their natural or their adoptive parents. Clearly until this number is reduced to an insignificant proportion of the total births no long-term plans can be made for the size of the population, and even people who doubt the need for such plans would agree that when one child in five is unwanted something ought to be done.

What is needed is a change in attitudes so that men and women of all ages see it as natural to approach doctors for advice on family planning, while doctors for their part should welcome these approaches and be able to give practical advice without embarrassment. At present few doctors have had formal training in contraceptive techniques. Some courses are run by the Family Planning Association and others by postgraduate medical centres, but undergraduate syllabuses are so crowded that contraception rarely finds the place it should. Another obstacle to the wider acceptance of advice on contraception as a natural part of the doctor's overall responsibility for the care of the family is the exclusion of the prescription of contraceptive appliances for "social" indications from the National Health Service.

Contraception still seems to be regarded in some quarters as a taboo subject. The select committee was told that "a lot of people will give sex education in schools, but if you want to mention contraception, this is not allowed." Unfortunately this attitude is found within the medical profession too. Pregnancy is still regarded by some as a natural retribution for sexual pleasure- 'you've had your fun, and now you've got to pay for it." In practice society pays for it in a legacy of disturbed, unhappy children struggling with an adverse environment. Pregnancy is not a good start to a marriage, and children in large families living in overcrowded housing conditions are at high risk of becoming undereducated delinquents. Yet contraceptive advice should be as much a part of preventive medicine as immunization against infectious diseases, and offered with the same expert knowledge and skill.

The Select Committee's report should encourage those responsible for undergraduate and postgraduate education to give higher priority to training in the field of family planning, 
for only when every child born is the result of a planned conception will it become clear whether there is a population problem at all.

1 Sclect Committce on Scicnce and Technology. First Rcport: Population of the United Kingdom. London, H.M.S.O., 1971.

\section{Choice of Penicillins for Gonorrhoea}

When considering the choice of penicillins to be administered there will always be some doctors and patients who are opposed to the treatment of uncomplicated gonorrhoea by injectible penicillin, and therefore it is right to review the efficacy of alternative oral treatment. Because so many patients default from surveillance immediately after treatment has relieved their symptoms, it is essential that effective oral treatment should be given in a single dose and that the cure rate be high.

Recently P. A. Kvale and colleagues ${ }^{1}$ reported that they had treated 510 United States naval ratings diagnosed as having gonorrhoea with various regimens of single-dosage oral penicillins. They gave a single dose of ampicillin $3.5 \mathrm{~g}$ to 68 patients, but there was a $29 \%$ failure rate in the 41 followed up. When probenecid $1.0 \mathrm{~g}$ was given to 373 patients one hour before the same dosage of ampicillin and then a further $0.5 \mathrm{~g}$ in 6,12 , and 18 hours, the failure rate was only $4 \%$ in the 202 followed up. On the other hand they found that oral phenoxymethyl penicillin, with dosage up to $7.5 \mathrm{~g}$ even with probenecid, gave failure rates ranging from 25 to $65 \%$ in the follow-up of 64 of the 69 patients treated. It is to be noted that in their ampicillin-probenecid regimen, though the patient takes the initial probenecid in the clinic, three further $0.5 \mathrm{~g}$ doses have to be taken later, and to this extent it cannot be regarded as single-dose therapy.

Most venereologists in the United Kingdom do not favour penicillin by mouth for gonorrhoea, and in this context a recent survey by the British Co-operative Clinical Group ${ }^{2}$ is of interest. A questionnaire was completed by 101 venereologists working in 206 clinics. Penicillin alone in various regimens was used in $79 \%$ and penicillin reinforced by other drugs in $20 \%$ of clinics. Ampicillin was reported to be used for males in five clinics and for females in 14 clinics. A majority of venereologists were not in favour of a moratorium on the use of penicillin with the idea of trying to reverse the decline in the sensitivity of the gonococcus to it.

When attempting to control gonorrhoea in a community every effort should certainly be made to prevent the development of resistant strains. The work of G. A. Olsen and G. Lomholt ${ }^{3}$ in Greenland represented a successful effort of this type. In 1963 they found in certain areas that there were as many as $86 \%$ (average $56 \%$ ) of partially resistant strains of gonococci, and the clinical failure rate with the penicillin regimen used was 26\%. Between 1964 and 1968 they treated over 800 patients with 5 megaunits of benzyl penicillin given in $0.5 \%$ lignocaine boosted by a single dose of probenecid $1.0 \mathrm{~g}$ (given 15 to 30 minutes before the injection). The cure rate was $99 \%$, and in the remaining $1 \%$ re-infection was the probable cause of the disease. By 1968 the percentage of partly resistant strains of gonococci had fallen to $19 \%$. Since then experience with this regimen in Britain has been equally satisfactory clinically. ${ }^{4}$

Thus treatment with injectible penicillin, preferably boosted by a single dose of probenecid, remains the most effective one-attendance therapy for gonorrhoea in this country, and oral ampicillin, which is more expensive and marginally less effective, must take second place.

It should be noted that in this brief discussion of the treatment of gonorrhoea with penicillin such alternative drugs as sulphamethoxazole-trimethoprim and kanamycin have not been considered, though they may be particularly indicated for patients with penicillin hypersensitivity or when early syphilis is suspected.

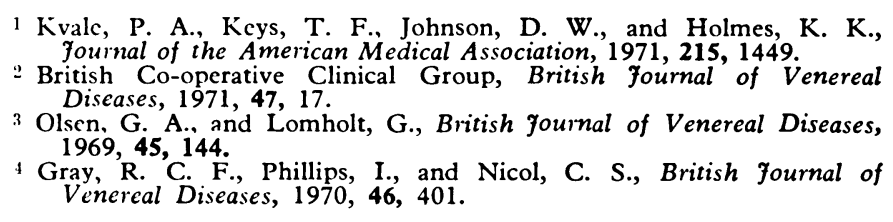

\section{Risks of Suddenly Stopping Anticoagulants}

Sudden cessation of treatment with heparin or warfarin, or its reversal with the antidotes protamine or vitamin $\mathrm{K}$, may be needed if a patient treated with an anticoagulant drug begins to bleed. Several reports have suggested that there is an increased risk of thrombosis or embolism if treatment is suddenly interrupted because of haemorrhage. ${ }^{1-3}$ The evidence on which these reports are based is open to criticism, but many physicians believe that there is a hypercoagulable "rebound" after stopping treatment and prefer to taper the dose off over several weeks.

Recent work suggests that the risks of stopping anticoagulant therapy suddenly may have been overstated. L. Michaels ${ }^{4}$ compared the incidence of thromboembolism in a group of 74 patients who had a sudden cessation of anticoagulant treatment because of haemorrhage with the experience of 166 others whose treatment had been stopped by choice. In 49 of the second group of patients, treatment had been tapered off, while it had been stopped abruptly in the remaining 117 . Clearly groups chosen in this way would not necessarily be comparable with one another, and Michaels endeavoured to overcome this objection by dividing his patients into three categories based on the estimated risk of recurrence. Using these criteria, he showed that the two groups of patients were sufficiently similar to allow comparison to be made. There was no difference in the risk of thromboembolism in the two groups either in total number of events or in their week-by-week incidence in the 16 weeks of follow-up after treatment was stopped. The risks of stopping treatment because of haemorrhage appeared to be no greater than if treatment was stopped electively.

There appears to be no need to hasten to restart anticoagulation (because of an assumed hazard of a hypercoagulable rebound) in a patient who has bled. The decision whether to restart treatment should be determined by balancing the risk of recurrent haemorrhage against the risk of recurrent thromboembolism. If the patient has a condition with a high risk of thromboembolism which will continue to operate after the drug has been stopped, it would be wise to restart anticoagulants as soon as it is safe to do so after 